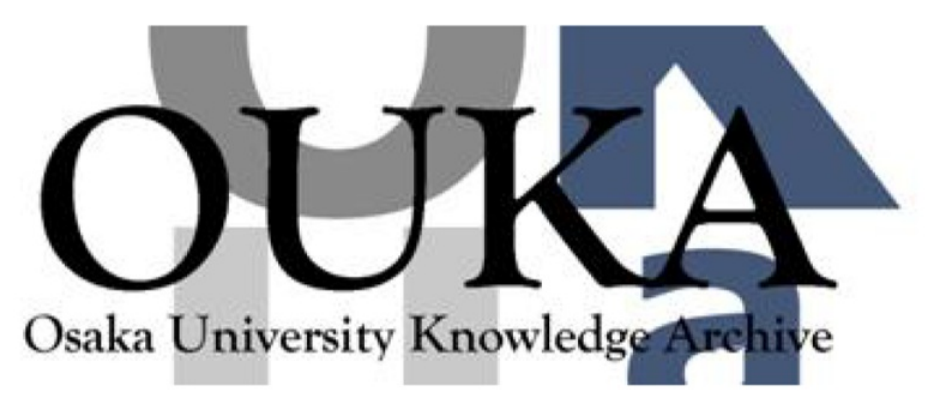

\begin{tabular}{|c|c|}
\hline Title & $\begin{array}{l}\text { Complete mode identification for resonance } \\
\text { ultrasound spectroscopy }\end{array}$ \\
\hline Author (s) & $\begin{array}{l}\text { Ogi, Hirotsugu; Sato, Keiji; Asada, Takeyasu et } \\
\text { al. }\end{array}$ \\
\hline Citation & $\begin{array}{l}\text { Journal of the Acoustical Society of America. } \\
112(6) \text { p. } 2553-p .2557\end{array}$ \\
\hline Issue Date & $2002-12$ \\
\hline oaire:version & VoR \\
\hline URL & https://hdl. handle. net/11094/84147 \\
\hline rights & $\begin{array}{l}\text { Copyright } 2002 \text { Acoustical Society of America. } \\
\text { This article may be downloaded for personal use } \\
\text { only. Any other use requires prior permission } \\
\text { of the author and the Acoustical Society of } \\
\text { America. }\end{array}$ \\
\hline Note & \\
\hline
\end{tabular}

Osaka University Knowledge Archive : OUKA

https://ir. Library. osaka-u. ac. jp/

Osaka University 


\title{
Complete mode identification for resonance ultrasound spectroscopy
}

\author{
Hirotsugu Ogi, ${ }^{a)}$ Keiji Sato, Takeyasu Asada, and Masahiko Hirao \\ Graduate School of Engineering Science, Osaka University, Machikaneyama 1-3, Toyonaka, \\ Osaka 560-8531, Japan
}

(Received 22 January 2002; revised 21 June 2002; accepted 9 August 2002)

\begin{abstract}
This study is devoted to deducing exact elastic constants of an anisotropic solid material without using any advance information on the elastic constants by incorporating a displacement-distribution measurement into resonant ultrasound spectroscopy (RUS). The usual RUS method measures free-vibration resonance frequencies of a solid and compares them with calculations to find the most suitable set of elastic constants by an inverse calculation. This comparison requires mode identification for the measured resonance frequencies, which has been difficult and never been free from ambiguity. This study then adopts a laser-Doppler interferometer to measure the displacement-distribution patterns on a surface of the vibrating specimen mounted on pinducers; comparison of the measured displacement distributions with those computed permits us to correctly identify the measured resonance frequencies, leading to unmistakable determination of elastic constants. Because the displacement patterns are hardly affected by the elastic constants, an exact answer is surely obtained even when unreasonable elastic constants are used as initial guesses at the beginning of the inverse calculation. The usefulness of the present technique is demonstrated with an aluminum alloy and a langasite crystal. (C) 2002 Acoustical Society of America.
\end{abstract}

[DOI: $10.1121 / 1.1512700]$

PACS numbers: 62.20.Dc, 43.20.Ks, 42.79.Qx [SGK]

\section{INTRODUCTION}

Resonant ultrasound spectroscopy (RUS) ${ }^{1-5}$ has been recognized as a useful method for determining all of the independent elastic constants $C_{i j}$ of an anisotropic solid in a regular shape such as a sphere, cylinder, or rectangular parallelepiped. The $C_{i j}$ are determined in two steps. First, a swept-frequency experiment measures many of the freevibration resonance frequencies of the specimen. Two transducers touch the specimen lightly, one for generation of a cw oscillation and the other for detection of the displacement amplitude. Second, an inverse calculation is performed to find the best fitting $C_{i j}$ that provide the closest resonance frequencies to the measurements. The resonance frequencies can be calculated using the specimen dimensions, mass density, and all of the $C_{i j}$.

The successful determination of elastic constants by the RUS method relies on exact correspondence between the observed and calculated resonance frequencies in the inverse calculation, that is, correct mode identification. If modes are incorrectly identified, the elastic constants may converge to a false minimum or fail to converge. However, mode identification has never been straightforward because the measured resonance spectrum contains a large number of resonance peaks, without showing any mode information. On the other hand, the calculation identifies the resonance modes. Thus, if one knows beforehand a set of elastic constants close to the true values, the calculation is highly likely to converge o the correct modes of the specimen. If one begins with very little information about the specimen's $C_{i j}$, it may not converge

${ }^{a)}$ Electronic mail: ogi@me.es.osaka-u.ac.jp correctly. Several efforts have been made to overcome this difficulty. Ohno ${ }^{2}$ noted different rates of changes of resonance frequencies when the specimen size varies. Maynard ${ }^{6}$ switched assignments of frequencies during the iteration calculation to find the best fit. Migliori et al. ${ }^{7}$ changed the specimen orientation relative to the transducers and monitored the change of signal amplitude. More recently, the present authors ${ }^{8,9}$ developed an electromagnetic-acoustic technique to select measurable vibration modes by controlling the electromagnetic-force direction. These methods are sometimes useful but still insufficient to make complete mode identification. They require supplementing RUS with some other methods such as pulse-echo and rod-resonance methods.

In this study, we propose an advanced methodology for this purpose. We adopt a laser-Doppler velocimeter to map a displacement-distribution pattern of a vibrating specimen. The displacements inside the specimen can be computed. Comparison of the measurements with the computations realizes correct mode identification and then the unmistakable determination of elastic constants.

\section{DISPLACEMENT MEASUREMENT AT RESONANCE}

Figure 1 shows the measurement setup of the RUS/Laser combination. A rectangular-parallelepiped specimen is put on a piezoelectric tripod consisting of two pinducers for generation and detection of vibration, and one for support. A $\mathrm{He}-\mathrm{Ne}$ laser beam is focused on the specimen surface (focal diameter: $15 \mu \mathrm{m}$ ) to scan the surface. The reflected beam enters the Doppler interferometer, which measures the nor- 


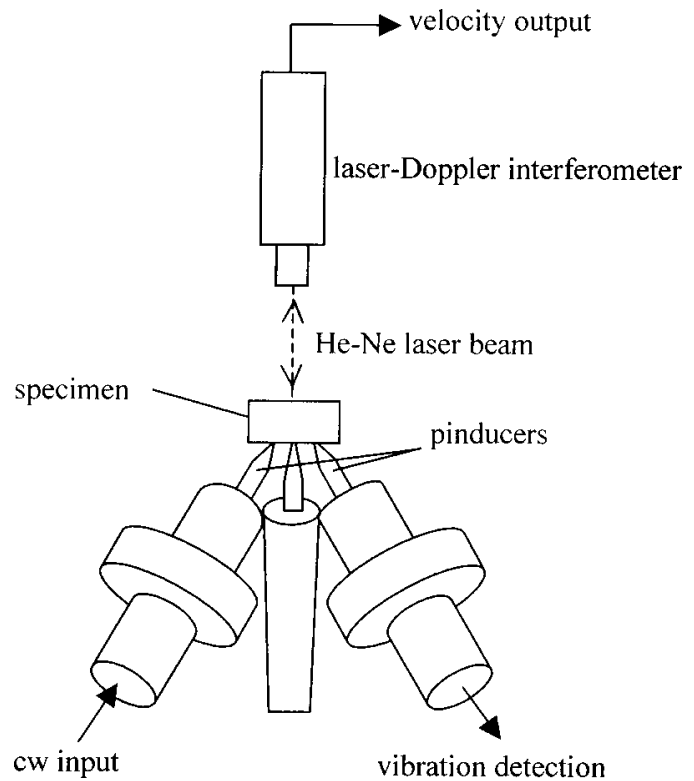

FIG. 1. RUS/Laser measurement setup.

mal component of the velocity at the focal point. The velocity is easily converted into the displacement because of harmonic oscillation.

First, we sweep the driving frequency to obtain the resonance spectrum as shown in Fig. 2 and measure the resonance frequencies by fitting a Lorentzian function around the peaks. The contact between the specimen and pinducers is weak and stable because only the specimen mass contributes to the acoustic coupling between the pinducers and specimen, which ensures high reproducibility in the resonancefrequency measurements. (Scattering of a measured resonance frequency was less than $10^{-4}$.)

Second, we drive one of the pinducers at a measured resonance frequency while scanning the specimen surface with the laser-Doppler interferometer to acquire the displacement distribution. The signal-to-noise ratio can be improved by Fourier-transforming the output signal from the interferometer to extract the component at the same frequency as the driving $\mathrm{cW}$ signal.

This RUS/Laser technique functioned for specimens with a mass larger than $0.01 \mathrm{~g}$, with a surface area larger than $2 \mathrm{~mm}$-by- $2 \mathrm{~mm}$ square, and with a $Q^{-1}$ value smaller than $10^{-3}$. Typical time needed to measure and display a displacement figure was $1 \mathrm{~min}$.

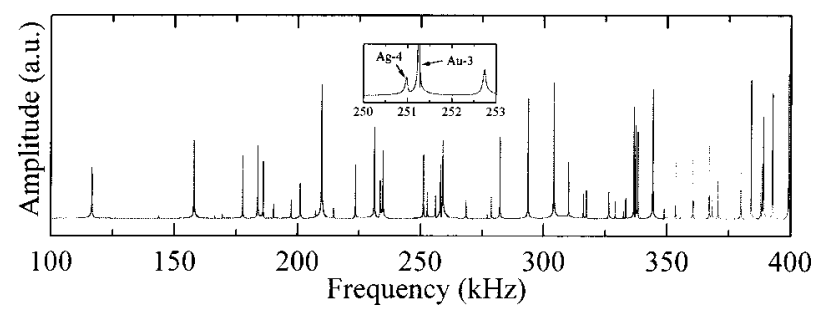

FIG. 2. Resonance spectrum measured by the pinducers for the aluminum alloy specimen. Only the specimen mass contributed to the dry acoustic coupling.

\section{CALCULATION OF DISPLACEMENT DISTRIBUTION}

No analytical solution exists for the displacements in a rectangular-parallelepiped solid subjected to a free vibration. Thus, the displacements have been approximated by linear combinations of basis functions, and the Rayleigh-Ritz approach has been adopted to determine the accompanying coefficients. Demarest ${ }^{1}$ showed that use of normalized Legendre functions as basis functions result in an accurate description of the displacements of a cube specimen with a minimum number of terms. Ohno ${ }^{2}$ applied a basis of Legendre functions to describe the displacements of a rectangular-parallelepiped specimen and established the calculation of the free-vibration resonance frequencies. We follow them in this study.

The displacement $u_{i}$ along the $x_{i}$ direction can be approximated as

$$
u_{i}\left(x_{1}, x_{2}, x_{3}\right)=\sum_{k} a_{k}^{(i)} \Psi_{k}^{(i)}\left(x_{1}, x_{2}, x_{3}\right)
$$

$$
\begin{aligned}
\Psi_{k}^{(i)}\left(x_{1}, x_{2}, x_{3}\right)= & \sqrt{\frac{8}{L_{1} L_{2} L_{3}}} \bar{P}_{1}\left(2 x_{1} / L_{1}\right) \\
& \times \bar{P}_{m}\left(2 x_{2} / L_{2}\right) \bar{P}_{n}\left(2 x_{3} / L_{3}\right),
\end{aligned}
$$

in a Cartesian coordinate system. $\bar{P}_{\lambda}$ denotes the normalized Legendre polynomial of degree $\lambda$ and $L_{i}$ denotes the edge length along the $x_{i}$ axis of the rectangular parallelepiped. Lagrangian minimization ${ }^{1-7}$ determines the free-vibration resonance frequencies and the coefficients $a_{k}^{(i)}$. The resonance frequencies have been compared with the measurements and the inverse calculation based on a least-squares fitting has inferred the $C_{i j}$.

There are eight vibration groups in the free vibration modes of a rectangular parallelepiped with orthorhombic symmetry, labeled as $A_{u}, A_{g}, B_{1 g}, B_{2 g}, B_{3 g}, B_{1 u}, B_{2 u}$, and $B_{3 u}$ by Mochizuki. ${ }^{10}$ Because the calculation of the resonance frequencies is independently performed for each vibration group, we exactly know the group and overtone order of the individual calculated frequencies, that is, we can completely identify them. On the other hand, the measured resonance frequencies never inform us of the mode information. Thus, incorrect comparison between the calculations and measurements, or mode misidentification, has easily occurred in the usual RUS method unless excellent initial guesses are adopted.

We pay attention to the coefficients $a_{k}^{(i)}$ to make correct mode identification. They tell us the two-dimensional pattern of the oscillating specimen surface, which is a signature of the individual mode. Thus, the correspondence between the measured and computed displacement distributions guarantees correct mode identification.

\section{RESULTS AND DISCUSSIONS}

\section{A. Polycrystalline aluminum alloy}

First, we demonstrate the capability of the present technique with a polycrystalline aluminum alloy. The specimen measures $L_{1}=11.92 \mathrm{~mm}, L_{2}=10.93 \mathrm{~mm}$, and $L_{3}=9.86 \mathrm{~mm}$ and has mass density $2788 \mathrm{~kg} / \mathrm{m}^{3}$. We assume isotropic elas- 


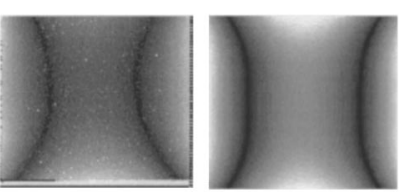

(a) $\mathrm{A}_{\mathrm{g}}-4(250.98 \mathrm{kHz})$
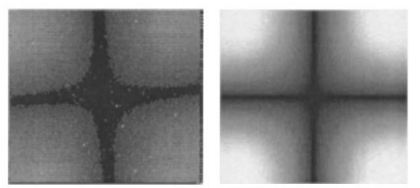

(b) $\mathrm{A}_{\mathrm{u}}-3(251.26 \mathrm{kHz})$

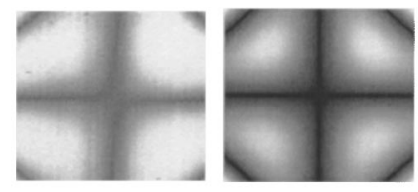

(c) $\mathrm{B}_{1 \mathrm{~g}}-5(336.65 \mathrm{kHz})$

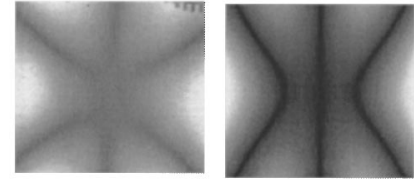

(d) $\mathrm{B}_{2 \mathrm{~g}}-5(337.36 \mathrm{kHz})$
FIG. 3. Comparison of the distribution of the normal displacement amplitude between measurements (left) and computations (right) for the aluminum alloy rectangular parallelepiped. The $x_{1}$ and $x_{2}$ axes are along the horizontal and vertical directions, respectively. The origin is located at the center of the rectangular parallelepiped.

tic symmetry and two independent elastic constants $C_{11}$ and $C_{44}$. Figure 2 shows the resonance spectrum measured by the piezoelectric tripod; many peaks appear and some of them overlap with each other. Thus, it is very difficult, as usual, to identify the modes without advance knowledge of the elastic constants. Figure 3 shows a comparison between the measured and computed displacement-distribution patterns. The computations used arbitrary elastic constants of $C_{11}=100$ and $C_{44}=20 \mathrm{GPa}$. Bright regions represent high amplitude displacements and dark regions represent low amplitude displacements; black means zero amplitude, the nodal lines. Typical maximum displacement was a few $\mathrm{nm}$ in magnitude. [We call these figures modern Chladni figures after Ernst F. F. Chladni (1756-1827), who visualized the resonance oscillations of a square brass plate by putting fine sand on it. ${ }^{11}$ ] We see very good agreement between the measurements and computations. The usefulness is remarkable, especially in identifying overlapping modes. For instance, the $A_{g}-4$ and $A_{u}-3$ resonances, indicated by arrows in Fig. 2 , occur at very close frequencies (only $0.1 \%$ difference) and it is hard to identify them. But, their displacement patterns are quite different from each other [Figs. 3(a) and (b)] and we can straightforwardly distinguish between them. Thus, by identifying all the observed modes (more than 80 now), we determined the $C_{i j}$ via the inverse calculation. Table I shows the measured and calculated resonance frequencies after convergence. Owing to the complete mode identification, there are no missed or extra modes in the measurement. The exact elastic constants are $C_{11}=109.26$ and $C_{44}=26.72 \mathrm{GPa}$. We tried to deduce the $C_{i j}$ using the usual RUS approach with the initial set of $C_{i j}$ used to compute the displacements in Fig. 3, and pairing the closest resonance frequencies from the measurements and calculations. The inverse calculation failed to converge, indicating that the usual RUS method is sensitive to the initial guesses.

Particularly significant is that the displacementdistribution patterns are insensitive to the elastic constants. In Fig. 4, we show the computed patterns for three cases; (I) isotropic symmetry with the exact $C_{i j}$; (II) isotropic symmetry with the $C_{i j}$ far away from the exact values; and (III) orthorhombic symmetry with arbitrary nine components of
TABLE I. Measured $\left(f_{\text {meas }}\right)$ and calculated $\left(f_{\text {calc }}\right)$ resonance frequencies $(\mathrm{kHz})$ after convergence for the aluminum alloy rectangular parallelepiped. Mode notation follows Mochizuki (Ref. 10). The average difference of $f_{\text {meas }}-f_{\text {calc }}$ was $0.2 \%$.

\begin{tabular}{|c|c|c|c|}
\hline Mode & $f_{\text {meas }}$ & $f_{\text {calc }}$ & Diff. $(\%)$ \\
\hline$A_{u}-1$ & 116.716 & 116.32 & 0.33 \\
\hline$A_{u}^{u}-2$ & 143.783 & 143.186 & 0.41 \\
\hline$B_{1 u}-1$ & 158.081 & 158.44 & -0.22 \\
\hline$B_{2 u}-1$ & 166.5 & 166.113 & 0.23 \\
\hline$B_{1 g}-1$ & 169.523 & 169.338 & 0.11 \\
\hline$B_{2 g}-1$ & 177.846 & 178.36 & -0.29 \\
\hline$B_{3 u}-1$ & 183.875 & 184.57 & -0.38 \\
\hline$B_{3 g}-1$ & 186.047 & 185.078 & 0.52 \\
\hline$A_{g}-1$ & 190.341 & 190.206 & 0.07 \\
\hline$B_{1 u}^{s}-2$ & 197.386 & 197.692 & -0.15 \\
\hline$A_{g}-2$ & 201.133 & 201.462 & -0.16 \\
\hline$B_{3 g}^{\circ}-2$ & 207.386 & 207.096 & 0.14 \\
\hline$A_{g}-3$ & 209.836 & 211 & -0.56 \\
\hline$B_{2 g}-2$ & 214.753 & 215.613 & -0.40 \\
\hline$B_{2 u}-2$ & 223.548 & 223.219 & 0.14 \\
\hline$B_{3 u}-2$ & 231.266 & 230.804 & 0.20 \\
\hline$B_{3 g}-3$ & 233.538 & 233.329 & 0.09 \\
\hline$B_{1 g}-2$ & 234.717 & 234.758 & -0.01 \\
\hline$A_{g}-4$ & 250.98 & 250.777 & 0.08 \\
\hline$A_{u}-3$ & 251.256 & 251.038 & 0.08 \\
\hline$B_{2 g}-3$ & 252.742 & 252.303 & 0.17 \\
\hline$B_{1 u}-3$ & 256.122 & 256.849 & -0.28 \\
\hline$B_{3 u}-3$ & 257.595 & 258.064 & -0.18 \\
\hline$A_{g}-5$ & 258.118 & 258.874 & -0.29 \\
\hline$B_{2 u}-3$ & 259.035 & 259.203 & -0.06 \\
\hline$B_{1 g}-3$ & 268.54 & 267.746 & 0.29 \\
\hline$B_{2 u}-4$ & 277.113 & 276.736 & 0.13 \\
\hline$B_{3 u}-4$ & 278.762 & 279.144 & -0.13 \\
\hline$B_{1 u}-4$ & 282.311 & 282.773 & -0.16 \\
\hline$B_{3 u}-5$ & 293.686 & 293.016 & 0.22 \\
\hline$B_{2 u}-5$ & 304.159 & 304.593 & -0.14 \\
\hline$B_{1 u}-5$ & 304.464 & 305.316 & -0.27 \\
\hline$B_{1 u}-6$ & 310.109 & 309.591 & 0.16 \\
\hline$B_{1 g}-4$ & 316.197 & 315.775 & 0.13 \\
\hline$B_{2 g}-4$ & 317.392 & 317.931 & -0.16 \\
\hline$A_{u}-4$ & 326.462 & 326.556 & -0.02 \\
\hline$B_{3 g}-4$ & 329.034 & 329.369 & -0.10 \\
\hline$A_{g}-6$ & 332.441 & 332.732 & -0.08 \\
\hline$B_{2 u}-6$ & 333.364 & 332.271 & 0.32 \\
\hline$B_{1 g}-5$ & 336.65 & 336.218 & 0.12 \\
\hline$B_{2 g}-5$ & 337.359 & 337.511 & -0.04 \\
\hline$A_{g}-7$ & 338.276 & 337.71 & 0.16 \\
\hline
\end{tabular}

$C_{i j}$. Despite the quite different $C_{i j}$, elastic symmetry, and accordingly resonance frequencies, the resemblance among the resultant patterns is striking, especially for the fundamental modes of the eight vibration groups. After all, such an oscillation pattern, or Chladni figure, is governed by the deformation symmetry allowed in the specimen. In the $\mathrm{Au}-$ group vibrations, for example, the $x_{3}$ component of displacement, $u_{3}$, occurs only when it is an odd function of $x_{1}$ and $x_{2} \cdot{ }^{2}$ Therefore, the Chladni figures on the $x_{3}$ face of this group always contain odd numbers of nodal lines running along each of the $x_{1}$ and $x_{2}$ axes. The fundamental mode, therefore, contains the cross-shaped node signature for every case. As for the $B_{3 g}$ group, $u_{3}$ must be an even function of $x_{1}$ and an odd function of $x_{2}$, so that the fundamental-mode Chladni figure shows one center nodal line along the $x_{1}$ axis; and so forth on. Thus, a Chladni figure is not directly related to the elastic constants. Note that the overtone order is not 


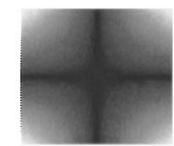

$\mathrm{A}_{\mathrm{u}}-1(116.3 \mathrm{kHz})$

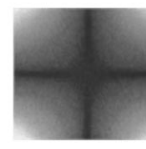

$\mathrm{A}_{\mathrm{u}}-1(159.1 \mathrm{kHz})$

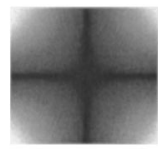

$A_{u}-1(129.3 \mathrm{kHz}) \quad A_{k}-1(177.6 \mathrm{kHz})$
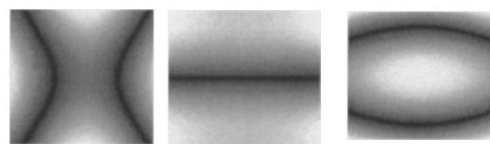

(a) case I
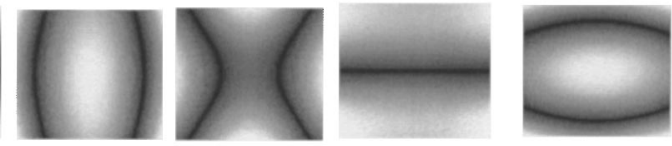

$\mathrm{A}_{\mathrm{g}}-1(260.4 \mathrm{kHz}) \mathrm{B}_{1 \mathrm{u}}-\mathrm{l}(218.0 \mathrm{kHz})$
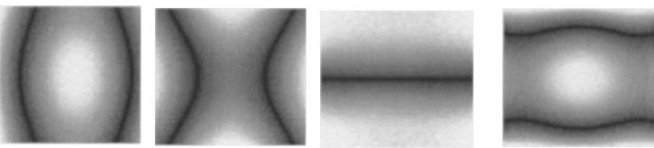

(c) case III

FIG. 4. Displacement-distribution patterns on the $x_{3}$ face for three cases: (a) isotropic symmetry with $C_{11}=109.26$ and $C_{44}=26.72 \mathrm{GPa}$; (b) isotropic symmetry with $C_{11}=300$ and $C_{44}=50 \mathrm{GPa}$; and (c) orthorhombic symmetry with $C_{11}=100, C_{22}=150, C_{33}=200, C_{12}=70, C_{13}=60, C_{23}=50, C_{44}$ $=20, C_{55}=30$, and $C_{66}=40 \mathrm{GPa}$. The $x_{1}$ and $x_{2}$ axes are along the horizontal and vertical directions, respectively. The origin is located at the center of the rectangular parallelepiped.

necessarily the same because the $C_{i j}$ affects the resonance frequencies to a large extent; for example, the Chladni figure for the third overtone of $A_{g}$ group $\left(A_{g}-3\right)$ in cases I and II appears at the fourth overtone in case III (see Fig. 4).

The mode-identification procedure thus becomes an easy task with this technique. Indeed, even unrealistic initial values of $C_{11}=300$ and $C_{44}=50 \mathrm{GPa}$ allowed us to make the complete mode identification and to reach the correct answer.

\section{B. Monocrystal langasite $\left(\mathrm{La}_{3} \mathrm{Ga}_{5} \mathrm{SiO}_{14}\right)$}

We applied this method to a transparent material, monocrystal langasite, by depositing a reflective thin film on the surface. Langasite is a candidate material for surfaceacoustic-wave filters owing to its large piezoelectric coefficients $e_{i j}$ and nearly temperature-independent elastic constants. The langasite's crystal structure belongs to the trigonal system with point group 32, thus involving six independent $C_{i j}$, two $e_{i j}$, and two dielectric coefficients $\kappa_{i j}$. All of them affect the free vibration, which means that $e_{i j}$ and $\kappa_{i j}$ can be determined by the RUS method together with the $C_{i j}$.

We used a rectangular parallelepiped crystal with dimensions of $L_{1}=8.027 \mathrm{~mm}, \quad L_{2}=9.804 \mathrm{~mm}, \quad$ and $L_{3}$ $=6.029 \mathrm{~mm}$. The mass density was $5725 \mathrm{~kg} / \mathrm{m}^{3}$. We deposited a 100 -nm-thick aluminum film on the surface normal to the $x_{3}$ axis (the three-fold axis of the trigonal system). The free-vibration modes for trigonal symmetry fall into the four groups of $A_{u}, A_{g}, B_{u}$, and $B_{g} \cdot{ }^{10}$ The Legendre basis functions in Eq. (2) are again available not only to describe the displacements but also to describe the electric potential. The detailed calculation procedure has been given by Ohno. ${ }^{12}$

We see excellent agreement between the measured and computed Chladni figures (Fig. 5). The crystal's elastic anisotropy causes asymmetric patterns about the $x_{1}$ axis (the two-fold axis). We could identify more than 80 observed resonance peaks, that is, up to more than 20 overtones in

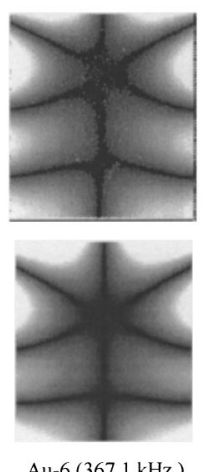

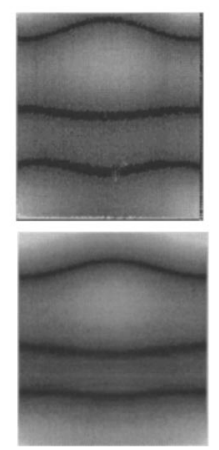

$\mathrm{Ag}-7(364.9 \mathrm{kHz})$

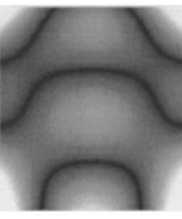

$\mathrm{Bu}-4(289.0 \mathrm{kHz})$

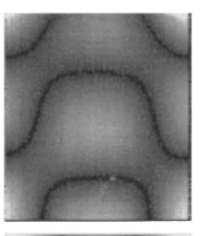

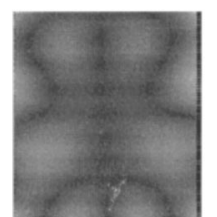

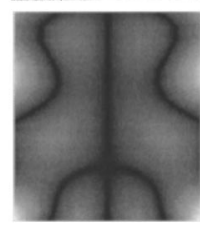

Bg-27 (676.5 kHz)
FIG. 5. Comparison of the displacement-distribution patterns on the $x_{3}$ face of the monocrystal langasite between measurements (upper) and computations (below). The $x_{1}$ and $x_{2}$ axes are along the horizontal and vertical directions, respectively. The origin is located at the center of the rectangular parallelepiped.

each group. Thus, the determination of all the material coefficients may be possible and we intend to report on this effort in near future.

\section{CONCLUSIONS}

We reached the following conclusions from this study.

(1) The RUS/Laser method presented here enables us to identify all observed vibration modes of a rectangular parallelepiped. This leads us to the exact elastic constants, removing the uncertainty in mode identification that has always troubled us.

(2) The displacement-distribution patterns are hardly affected by the elastic constants and elastic symmetry. Therefore, by comparing the measured and computed displacement patterns, we can obtain exact elastic constants with initial guesses far away from the truth. Even the initial values more than $200 \%$ different from the correct values resulted in the exact answer.

(3) The present technique also applies to a transparent material with a reflective thin film deposited on the specimen surface. This was demonstrated using a monocrystal langasite.

\section{ACKNOWLEDGMENTS}

Gratefully, we acknowledge Takekazu Miya (Sonix K. K.) and Dr. Tetsu Ichitsubo (Osaka University) for giving us valuable comments.

${ }^{1}$ H. Demarest, Jr., "Cube resonance method to determine the elastic constants of solids,” J. Acoust. Soc. Am. 49, 768-775 (1971).

${ }^{2}$ I. Ohno, "Free vibration of a rectangular parallelepiped crystal and its application to determination of elastic constants of orthorhombic crystals," J. Phys. Earth 24, 355-379 (1976).

${ }^{3}$ J. Maynard, "Resonant ultrasound spectroscopy," Phys. Today 49, 26-31 (1996).

${ }^{4}$ A. Migliori and J. Sarrao, Resonant Ultrasound Spectroscopy (Wiley, New York, 1997).

${ }^{5}$ H. Ledbetter, C. Fortunko, and P. Heyliger, "Orthotropic elastic constants of a boron-aluminum fiber-reinforced composite: An acoustic-resonancespectroscopy study," J. Appl. Phys. 78, 1542-1546 (1995).

${ }^{6} \mathrm{~J}$. Maynard, "The use of piezoelectric film and ultrasound resonance to 
determine the complete elastic tensor in one measurement," J. Acoust. Soc. Am. 91, 1754-1762 (1992).

${ }^{7}$ A. Migliori, J. Sarrao, M. W. Visscher, T. Bell, M. Lei, Z. Fisk, and R. Leisure, "Resonant ultrasound spectroscopy techniques for measurement of the elastic moduli of solids," Physica B 183, 1-24 (1993).

${ }^{8}$ H. Ogi, H. Ledbetter, S. Kim, and M. Hirao, "Contactless mode-selective resonance ultrasound spectroscopy: Electromagnetic acoustic resonance," J. Acoust. Soc. Am. 106, 660-665 (1999).

${ }^{9}$ H. Ogi, K. Takashima, H. Ledbetter, M. L. Dunn, G. Shimoike, M. Hirao, and P. Bowen, "Elastic constants and internal friction of an SiC-fiber- reinforced Ti-alloy-matrix crossply composite: Measurement and theory," Acta Mater. 47, 2787-2796 (1999).

${ }^{10} \mathrm{E}$. Mochizuki, "Application of group theory to free oscillations of an anisotropic rectangular parallelepiped," J. Phys. Earth 35, 159-170 (1987).

${ }^{11}$ C. Taylor, The Art and Science of Lecture Demonstration (IOP, Bristol, 1995).

${ }^{12}$ I. Ohno, "Rectangular parallelepiped resonance method for piezoelectric crystals and elastic constants of alpha-quartz," Phys. Chem. Miner. 17, 371-378 (1990). 